\title{
Stimulated emission of near-infrared radiation in silicon fin light-emitting diode
}

\author{
S. Saito, ${ }^{1,2,3, a)}$ T. Takahama,${ }^{3}$ K. Tani, ${ }^{1,2,3}$ M. Takahashi, ${ }^{1,2,3}$ T. Mine,${ }^{3}$ Y. Suwa, ${ }^{1,2,3}$ \\ and D. Hisamoto ${ }^{3}$ \\ ${ }^{1}$ Institute for Photonics-Electronics Convergence System Technology (PECST), Tokyo 185-8601, Japan \\ ${ }^{2}$ Photonics Electronics Technology Research Association (PETRA), Tokyo 185-8601, Japan \\ ${ }^{3}$ Central Research Laboratory, Hitachi, Ltd., Kokubunji, Tokyo 185-8601, Japan
}

(Received 10 May 2011; accepted 8 June 2011; published online 28 June 2011)

\begin{abstract}
We propose top-down processes to make silicon multiple quantum wells called fins for a lightemitting diode. The silicon fins are formed vertically to a substrate and embedded in a $\mathrm{Si}_{3} \mathrm{~N}_{4}$ waveguide. By current injections into silicon fins, we have observed stimulated emission spectra peaked at the wavelengths corresponding to the periodic structures of fins. The near-field mode profiles obtained at the edge of the waveguide qualitatively agreed with theoretical calculations. It has been turned out that both transverse-electric and transverse-magnetic fields can contribute to the optical gain. (C) 2011 American Institute of Physics. [doi:10.1063/1.3605255]
\end{abstract}

Quantum mechanical confinements of semiconductor nanomaterials drastically change their physical properties from those in the bulk. ${ }^{1}$ In silicon $(\mathrm{Si})$, the confinements change its band structure from indirect to direct one, making efficient radiative recombinations possible. ${ }^{2-7}$ In fact, the stimulated emissions were observed in Si nanocrystals by optical pumping. ${ }^{8}$ To make these nanostructures, the bottom-up processes were usually employed such as electro-chemical etching, ${ }^{2,3}$ molecular beam expitaxy (MBE), ${ }^{4,5}$ or annealing of $\mathrm{Si}$ implanted $\mathrm{SiO}_{2}$. While the electro-chemical etching of sigle crystal $\mathrm{Si}^{2,3,9}$ to make porous $\mathrm{Si}$ is rather close to the top-down approach, fabrication processes must be carefully monitored to control the size of nanostructures ${ }^{9}$ and the chemical bonding configurations for the surface passivation. ${ }^{10}$ On the other hand, the rapid progress of the Si nanoelectronics enables us to make such structures by top-down processes. ${ }^{11-18}$ By the thermal oxidation of silicon-on-insulator (SOI) substrates, $\mathrm{Si}$ single quantum wells (SQWs) were formed and the excellent $\mathrm{Si} / \mathrm{SiO}_{2}$ interfacial quality were confirmed by photoluminescence. ${ }^{11-13}$ The electroluminecence (EL) ${ }^{14-18}$ and stimulated emissions ${ }^{17}$ were also demonstrated by lateral current injections into Si SQWs.

The next requirement towards a practical Si laser diode for $\mathrm{Si}$ photonics ${ }^{6}$ is to enhance the effective gain value obtained by the evanescent coupling between the guided optical mode and the Si SQW. ${ }^{17}$ The obvious approach is to increase the number of QWs from SQW to multiple quantum wells (MQWs). However, it is not easy to deposit Si MQWs whose surface was covered with amorphous $\mathrm{SiO}_{2}$ by epitaxial growth. ${ }^{19}$ Unlike compound semiconductor technologies, vertical stacks of MQWs are difficult to make in planar $\mathrm{Si}$ processes. The purpose of this work is to fabricate Si MQWs by standard top-down Si processes and examine their potential abilities as a monolithic light source integrated on a chip towards the convergence of electronics and photonics.

The proposed Si fin light-emitting diode (FinLED) is shown in Fig. 1(a). Instead of a lateral SQW, MQWs are

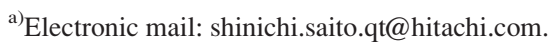

formed perpendicular to the substrate. Each side of MQWs is connected with a heavily doped Si electrode, forming a lateral pin diode. In this FinLED, thousands of QWs can be integrated by simple lithography and etching processes. The similar multi-fin device structure known as a FinFET was proposed as a double gate field-effect-transistor (FET). ${ }^{20}$ The differences between a FinLED and a FinFET are impurity profiles and absence/presence of gate processes. Therefore, it is possible to integrate both devices solely from the small modification of process steps. The practical challenge for the FinLED is that the width of fins should be sufficiently small to expect the efficient light emission by quantum confinements, while for the FinFET, the width should be larger to avoid the threshold voltage increase.

The fabricated Si FinLED is shown in Fig. 1(b). The fin with the width of $20 \mathrm{~nm}$ and the height of $50 \mathrm{~nm}$ was formed

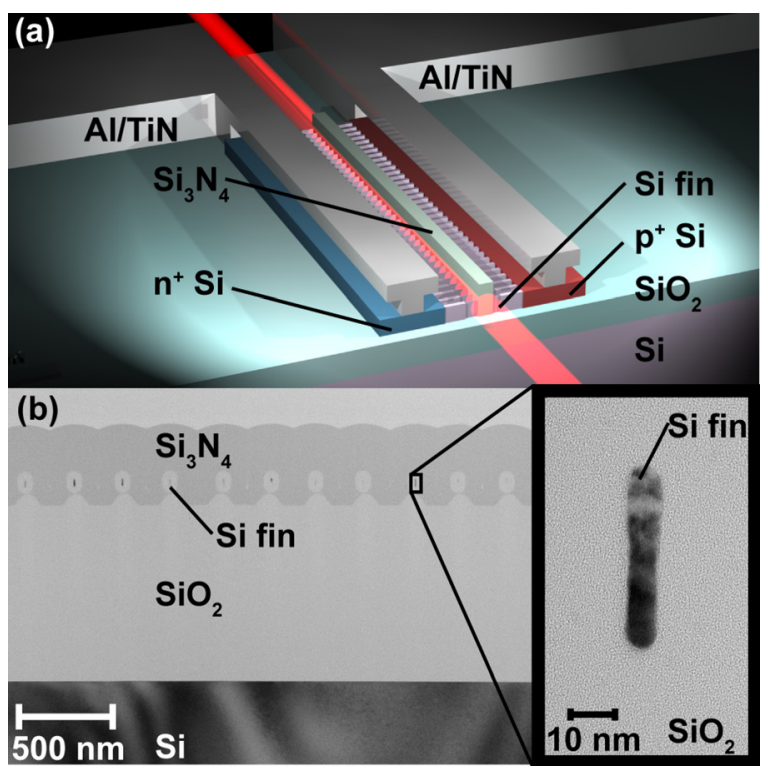

FIG. 1. (Color online) Si FinLED. (a) Schematic device structures. Active channel width (length) is 500 (1) $\mu \mathrm{m}$, and cavity length (width) is $500(0.6)$ $\mu \mathrm{m}$. (b) Transmission electron microscope (TEM) images after processing. Inset shows single $\mathrm{Si}(100)$ fin. 

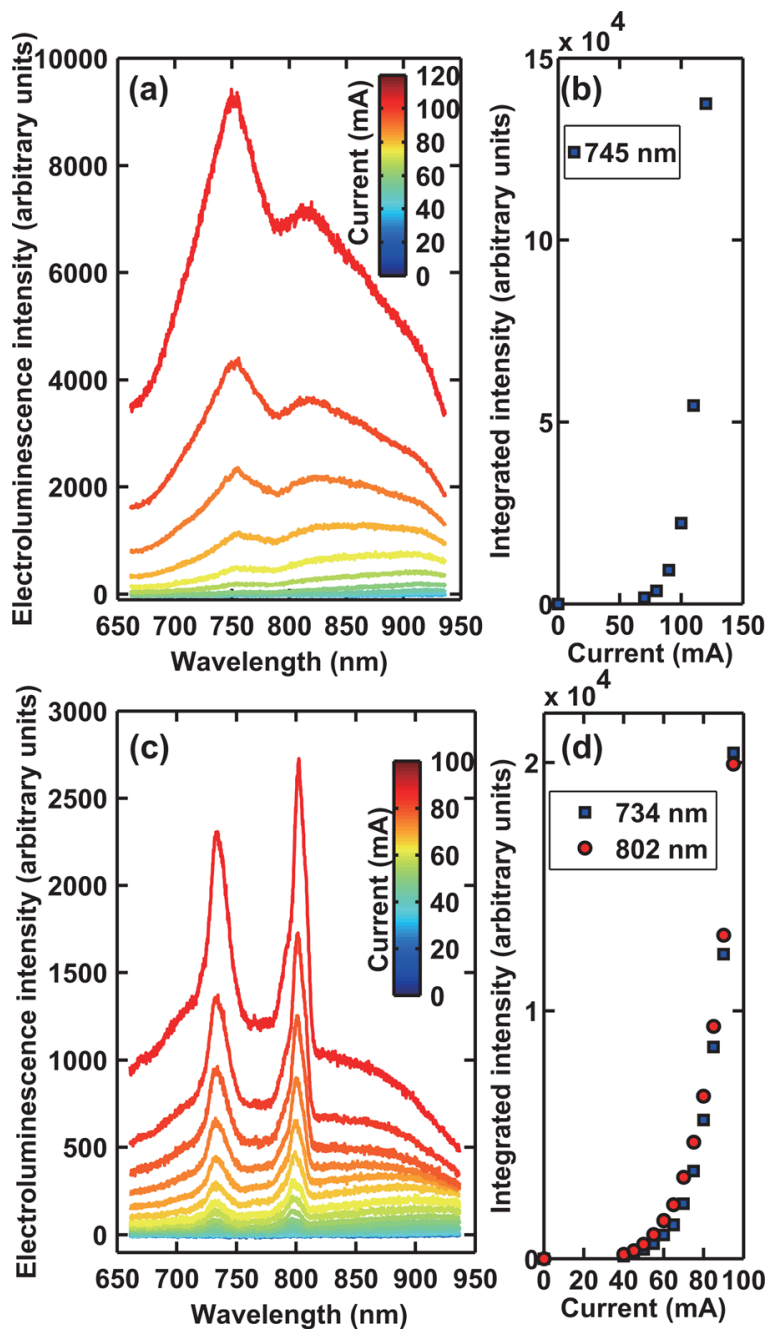

FIG. 2. (Color online) Stimulated emissions from Si FinLED. EL spectra from FinLED with $\Lambda$ of (a) $230 \mathrm{~nm}$ and (c) $300 \mathrm{~nm}$. Single and multiple peaks dominated at $745.1 \mathrm{~nm}$ in (a) and at 734.4 and $802.4 \mathrm{~nm}$ in (c). Peak intensity from FinLED with $\Lambda$ of (b) $230 \mathrm{~nm}$ and (d) $300 \mathrm{~nm}$.

after the dry etching, and the width was further reduced by the subsequent oxidation processes down to $5 \mathrm{~nm}$ or below. We prepared two devices with different fin pitch $\Lambda$ of 230 $\mathrm{nm}$ and $300 \mathrm{~nm}$ for comparisons. After the formation of multi-fins, the heavily doped electrodes are formed by ion implantations and the activation. Then, the $\mathrm{Si}_{3} \mathrm{~N}_{4}$ film with the thickness of $250 \mathrm{~nm}$ was deposited by a chemical-vapordeposition process and patterned to become a core of a waveguide located at the center of Si fins. The cavity length $L_{\text {cavity }}$ is $500 \mu \mathrm{m}$. After the metallization by $\mathrm{Al} / \mathrm{TiN}$, the $\mathrm{H}_{2}$ annealing is performed to passivate the interface traps. All optical and electrical measurements were made by constant current conditions at room temperatures.

The EL spectra taken from the edge of the $\mathrm{Si}_{3} \mathrm{~N}_{4}$ waveguide of Si FinLEDs are shown in Figs. 2(a) and 2(c). The emitted wavelengths were wide range from $600 \mathrm{~nm}$ to $1000 \mathrm{~nm}$, suggesting there are a few nm variations of the widths of the fin due to the line-edge roughness as well as the variations among different fins due to process fluctuations. The maximum of the broad spectrum background of $\sim 750 \mathrm{~nm}$ corresponds to emissions from Si fins with widths of around $1.0 \mathrm{~nm}$ according to our theoretical calculations. ${ }^{7}$ Several peaks are observed in the spectra, and the peak wavelengths are different between devices with different $\Lambda$. By extracting the peaks by the Gaussian forms, we have found that the peaks developed super-linearly with injected currents, as shown in Figs. 2(b) and 2(d). These data indicates that stimulated emissions from $\mathrm{Si}$ fins have been induced under high currents. ${ }^{17}$ On the other hand, the emissions from the broad background also developed after the onset of the peaks, and therefore, the maximum current levels are close to but still below the lasing thresholds. Increasing the currents further broke the devices, and the devices become completely open, suggesting chain reactions at the breakdown.

In order to understand these spectral peaks, we observed near field beam profiles at the edge of the waveguide and compared with theoretical calculations as shown in Fig. 3. In the FinLED with $\Lambda$ of $230 \mathrm{~nm}$ (Fig. 3(a)), the lowest cavity modes of $\mathrm{TE}_{00}$ (Fig. 3(c)) and $\mathrm{TM}_{00}$ (Fig. 3(h)) with the higher effective index $n_{0}$ were dominated. Both transverseelectric (TE) and transverse-magnetic (TM) fields can contribute, since MQWs of Si fins are located perpendicularly to the optical propagation direction in the waveguide. On the other hand, in the FinLED with $\Lambda$ of $300 \mathrm{~nm}$ (Fig. 3(b)), the near field image shows that the beam spreads vertically to the substrate, although the horizontal width of the waveguide $(600 \mathrm{~nm})$ is much larger than the thickness $(250 \mathrm{~nm})$. Therefore, the higher modes spreading on the buried-oxide (BOX) layer would be responsible.

For each propagating mode, we have estimated stimulated emission wavelength $\lambda_{\text {th }}$ by an index and gain coupled distributed-feedback (DFB) structure model. ${ }^{21}$ Assuming the refractive index, $n=n_{0}+n_{1} \cos (2 \pi z / \Lambda)$, and the gain, $g=g_{0}+g_{1} \cos (2 \pi z / \Lambda)$, are modulated along the cavity $z$, the lasing threshold condition becomes
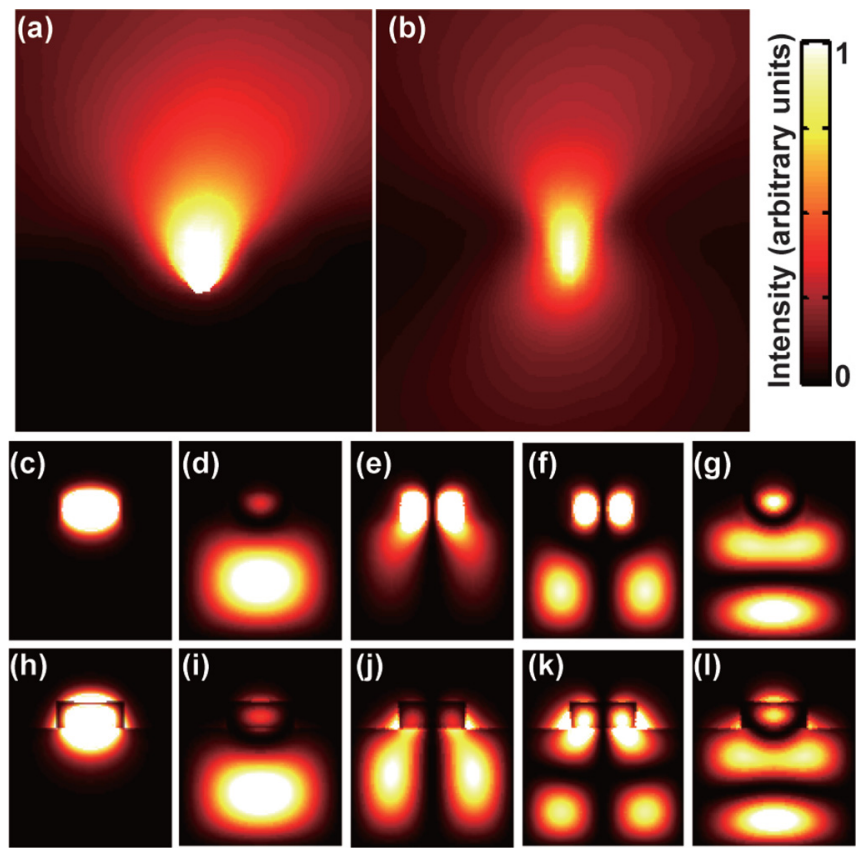

FIG. 3. (Color online) Near-field images from Si FinLED. Experimental EL images from FinLED with $\Lambda$ of (a) $230 \mathrm{~nm}$ and (b) $300 \mathrm{~nm}$. Images were obtained with integration time of $10 \mathrm{~s}$ under constant currents of $100 \mathrm{~mA}$ and $85 \mathrm{~mA}$ for $\Lambda$ of $230 \mathrm{~nm}$ and $300 \mathrm{~nm}$. Calculated mode profiles for (c) $\mathrm{TE}_{00}$, (d) $\mathrm{TE}_{01}$, (e) $\mathrm{TE}_{10}$, (f) $\mathrm{TE}_{11}$, (g) $\mathrm{TE}_{02}$, (h) $\mathrm{TM}_{00}$, (i) $\mathrm{TM}_{01}$, (j) $\mathrm{TM}_{10}$, (k) $\mathrm{TM}_{11}$, and (1) $\mathrm{TM}_{02}$. 
TABLE I. Calculated device parameters of FinLED for various propagation modes.

\begin{tabular}{llllll}
\hline \hline Parameters & $\mathrm{TE}_{00}$ & $\mathrm{TE}_{01}$ & $\mathrm{TE}_{10}$ & $\mathrm{TE}_{11}$ & $\mathrm{TE}_{02}$ \\
\hline$n_{0}$ & 1.682 & 1.388 & 1.376 & 1.299 & 1.275 \\
$n_{1}$ & 0.016 & 0.002 & 0.017 & 0.006 & 0.008 \\
$\kappa_{1} L_{\text {cavity }}$ & 24.8 & 3.92 & 32.3 & 11.7 & 17.1 \\
$\lambda_{\text {th }}[\mathrm{nm}]^{\mathrm{a}}$ & 774.1 & 639.3 & 627.8 & 595.7 & 584.0 \\
$\lambda_{\text {th }}[\mathrm{nm}]^{\mathrm{b}}$ & 1013.3 & 833.9 & 818.9 & 776.9 & 761.8 \\
$g_{1 \text { th }}\left[\mathrm{cm}^{-1}\right]^{\mathrm{a}}$ & 0.8 & 26.2 & 0.4 & 3.3 & 1.6 \\
$g_{1 \text { th }}\left[\mathrm{cm}^{-1}\right]^{\mathrm{b}}$ & 1.27 & 40.4 & 0.8 & 5.6 & 2.7 \\
\hline Parameters & $\mathrm{TM}_{00}$ & $\mathrm{TM}_{01}$ & $\mathrm{TM}_{10}$ & $\mathrm{TM}_{11}$ & $\mathrm{TM}_{02}$ \\
\hline$n_{0}$ & 1.557 & 1.380 & 1.336 & 1.255 & 1.254 \\
$n_{1}$ & 0.022 & 0.001 & 0.009 & 0.017 & 0.004 \\
$\kappa_{1} L_{\text {cavity }}$ & 38.3 & 1.4 & 17.9 & 8.5 & 36.3 \\
$\lambda_{\text {th }}[\mathrm{nm}]^{\mathrm{a}}$ & 720.3 & 634.9 & 616.0 & 577.3 & 580.1 \\
$\lambda_{\text {th }}[\mathrm{nm}]^{\mathrm{b}}$ & 939.6 & 828.1 & 803.5 & 753.0 & 756.6 \\
$g_{1 \text { th }}\left[\mathrm{cm}{ }^{-1}\right]^{\mathrm{a}}$ & 0.3 & 91.0 & 1.4 & 6.2 & 0.4 \\
$g_{1 \text { th }}\left[\mathrm{cm}^{-1}\right]^{\mathrm{b}}$ & 0.5 & 103.7 & 2.4 & 10.3 & 0.6 \\
\hline \hline
\end{tabular}

${ }^{\mathrm{a} C}$ Calculated for $\Lambda=230 \mathrm{~nm}$.

${ }^{\mathrm{b} C}$ Calculated for $\Lambda=300 \mathrm{~nm}$.

$$
s \cosh \left(s L_{\text {cavity }}\right)+i\left(\delta \beta+\frac{i g_{0}}{2}\right) \sinh \left(s L_{\text {cavity }}\right)=0,
$$

where $s=\sqrt{\left(\kappa_{1} L_{\text {cavity }}-i g_{1} L_{\text {cavity }} / 4\right)^{2}-(\delta \beta)^{2}}, \kappa_{1}=\pi n_{1} / \lambda_{0}$, the Bragg wavelength $\lambda_{0}=2 n_{0} \Lambda$, and the deviation $\delta \beta$ from the Bragg vector $\beta=2 \pi / \lambda_{0}$. $\lambda_{\text {th }}$ is the longer wavelength of the stop band edges and obtained from a solution of Eq. (1). The calculated parameters including required threshold gain value $g_{1 \text { th }}$ for lasing are summarized in Table I. For the FinLED with $\Lambda$ of $300 \mathrm{~nm}, \mathrm{TM}_{10}$ and $\mathrm{TM}_{11}$ are the most probable modes to explain the observed spectra and near field images. But, the other modes like $\mathrm{TE}_{10}$ and $\mathrm{TE}_{11}$ can contribute to the peaks, and the broad spontaneous emissions can be propagated by $\mathrm{TE}_{00}$ and $\mathrm{TM}_{00}$. The calculated wavelengths qualitatively agreed with experimental ones, regardless of neglecting the optical leakage to the substrate in higher modes. These multiple modes would be responsible to explain the spectra of Fig. 2(c) and the observed mode profile of Fig. 3(b).

We have proposed a Si fin light-emitting diode to realize multiple quantum wells fabricated by top-down $\mathrm{Si}$ processes. The $\mathrm{Si}$ fins embedded in a $\mathrm{Si}_{3} \mathrm{~N}_{4}$ waveguide worked as an index and gain coupled distributed-feedback structure. By lateral current injections into Si fins, stimulated emissions of near-infrared radiations have been observed at the edge of the $\mathrm{Si}_{3} \mathrm{~N}_{4}$ waveguide. Si based light emitters should open up a new opportunity for the convergence of photonics and electronics on a Si chip.

We thank Professor Y. Arakawa, Professor S. Iwamoto, Dr. S. Kako, M. Nakamura, T. Onai, N. Osakabe, K. Torii, T. Ido, M. Aoki, S. Kimura, T. Sugawara, H. Arimoto, E. Nomoto, R. Tsuchiya, Y. Matsui, H. Yoshimoto, I. Yanagi, and T. Arai for enlightening discussions. We also thank K. Takeda, J. Yamamoto, Y. Matsuoka, T. Takahashi, I. Uchida, S. Morita, T. Morimoto, M. Kawano, T. Yamamoto, H. Ichizoe, K. Endo, N. Oshima, N. Higuchi, H. Hamamura, K. Fujisaki, and K. Hozawa for helping in device fabrications. This research is granted by the Japan Society for the Promotion of Science (JSPS) through the "Funding Program for World-Leading Innovative R\&D on Science and Technology (FIRST Program)," initiated by the Council for Science and Technology Policy (CSTP).

${ }^{1}$ Y. Arakawa and H. Sakaki, Appl. Phys. Lett. 40, 939 (1982).

${ }^{2}$ L. T. Canham, Appl. Phys. Lett. 57, 1046 (1990).

${ }^{3}$ N. Koshida and H. Koyama, Appl. Phys. Lett. 60, 347 (1991).

${ }^{4}$ Z. H. Lu, D. J. Lockwood, and J. M. Baribeau, Nature 378, 258 (1995).

${ }^{5}$ D. J. Lockwood, Z. H. Lu, and J. M. Baribeau, Phys. Rev. Lett. 76, 539 (1996).

${ }^{6}$ S. Ossicini, L. Pavesi, and F. Priolo, Light Emitting Silicon for Microphotonics (Springer, Berlin, 2003).

${ }^{7}$ Y. Suwa and S. Saito, Phys. Rev. B 79, 233308 (2009).

${ }^{8}$ L. Pavesi, L. D. Negro, C. Mazzoleni, G. Franzò, and F. Priolo, Nature 408, 440 (2000).

${ }^{9}$ A. G. Cullis, L. T. Canham, and P. D. J. Calcott, J. Appl. Phys. 82, 909 (1997).

${ }^{10}$ B. Gelloz, A. Kojima, and N. Koshida, Appl. Phys. Lett. 87, 031107 (2005).

${ }^{11}$ Y. Takahashi, T. Furuta, Y. Ono, T. Ishiyama, and M. Tabe, Jpn. J. Appl. Phys. 34, 950 (1995).

${ }^{12}$ Z. H. Lu and D. Grozea, Appl. Phys. Lett. 80, 255 (2002).

${ }^{13}$ N. Pauc, V. Calvo, J. Eymery, F. Fournel, and N. Magnea, Phys. Rev. Lett. 92, 236802 (2004).

${ }^{14}$ S. Saito, D. Hisamoto, H. Shimizu, H. Hamamura, R. Tsuchiya, Y. Matsui, T. Mine, T. Arai, N. Sugii, K. Torii, S. Kimura, and T. Onai, Jpn. J. Appl. Phys. 45, L679 (2006).

${ }^{15}$ S. Saito, D. Hisamoto, H. Shimizu, H. Hamamura, R. Tsuchiya, Y. Matsui, T. Mine, T. Arai, N. Sugii, K. Torii, S. Kimura, and T. Onai, Appl. Phys. Lett. 89, 163504 (2006).

${ }^{16}$ T. Hoang, P. LeMinh, J. Holleman, and J. Schmitz, IEEE Electron Device Lett. 28, 383 (2007).

${ }^{17}$ S. Saito, Y. Suwa, H. Arimoto, N. Sakuma, D. Hisamoto, H. Uchiyama, J. Yamamoto, T. Sakamizu, T. Mine, S. Kimura, T. Sugawara, and M. Aoki, Appl. Phys. Lett. 95, 241101 (2009).

${ }^{18}$ J. Noborisaka, K. Nishiguchi, Y. Ono, H. Kageshima, and A. Fujiwara, Appl. Phys. Lett. 98, 033503 (2011).

${ }^{19}$ L. Ding, M. B. Yu, X. Tu, G. Q. Lo, S. Tripathy, and T. P. Chen, Opt. Lett. 19, 2729 (2011).

${ }^{20}$ D. Hisamoto, W. C. Lee, J. Kedzierski, H. Takeuchi, K. Asano, C. Kuo, E. Anderson, T. J. King, J. Bokor, and C. Hu, IEEE Trans. Electron Devices 47, 2320 (2000).

${ }^{21}$ A. Yariv and P. Yeh, Photonics: Optical Electronics in Modern Communications (Oxford University Press, New York, 2007). 